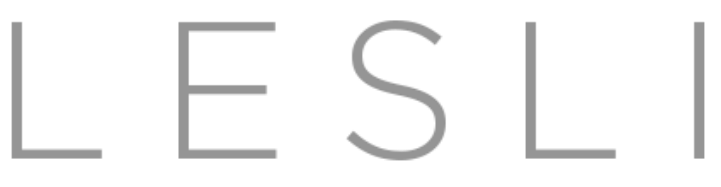

Linguistic Evidence in Security, Law and Intelligence

Volume 1, No. 1 (2013) | ISSN 2327-5596 (online) | DOI 10.5195/lesli.2013.6 | http://lesli-journal.org

\title{
Detection of Deception in a Virtual World
}

\author{
Lauren B. Collister PhD \\ University of Pittsburgh; Research Associate, Institute for Linguistic Evidence, USA \\ $\underline{\text { lbc8@ @itt.edu }}$
}

\begin{abstract}
This work explores the role of multimodal cues in detection of deception in a virtual world, an online community of World of Warcraft players. Case studies from a five-year ethnography are presented in three categories: small-scale deception in text, deception by avoidance, and large-scale deception in game-external modes. Each case study is analyzed in terms of how the affordances of the medium enabled or hampered deception as well as how the members of the community ultimately detected the deception. The ramifications of deception on the community are discussed, as well as the need for researchers to have a deep community knowledge when attempting to understand the role of deception in a complex society. Finally, recommendations are given for assessment of behavior in virtual worlds and the unique considerations that investigators must give to the rules and procedures of online communities.
\end{abstract}

Keywords: deception detection, online community, virtual world

\section{Introduction}

Peter Steiner's adage about the Internet age, "On the Internet, nobody knows you're a dog," suggests that an affordance of online mediums is deception about identity. Whether this affordance provides an emancipatory environment where freedom of expression is unlimited and licensed (Jordan, 1999, p.66) or a breeding ground for deception and false connections (Trend, 2001, p.226) has been a topic of much theoretical debate; however, relatively little work has been done to show how the deceived parties detect inauthentic identity portrayals in online contexts. In this work, I will provide examples of identity deception from an ethnographic study of a community of World of Warcraft players. First, I will describe the game environment and how the study was performed; then, I will describe three different categories of deception defined both by the intent of the deceiver and the linguistic mode in which the deception occurs. I will follow this by describing the methods by which members of the community detected deception in each of these three categories. This case study highlights the ways that a

\section{$(\mathrm{cc}) \mathrm{Er}$}

Articles in this journal are licensed under a Creative Commons Attribution 3.0 United States License.

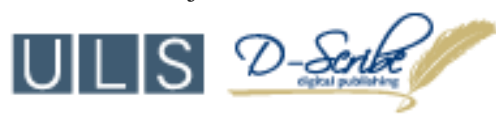

This journal is published by the University Library System, University of Pittsburgh as part of its D-Scribe

Digital Publishing Program and is cosponsored by the University of Pittsburgh Press. 
multimodal approach can offer a broader base of cues from which to detect identity deception, as well as illustrating the benefits of a deep understanding of the role of deception in community norms and practices.

\section{The Game}

The field site for this study is World of Warcraft, a Massively Multiplayer Online Role-Playing Game (MMORPG). At the time of this article, World of Warcraft is the most popular game of its type in the world with a 7.6 million subscribers and had a peak of 12 million subscribers in $2010 .{ }^{1}$ To play the game, an individual creates an account on the game's website, downloads the game client onto their computer, and logs in to create the avatar which will be their presence in the game world. Avatars come from a variety of fantasy races (e.g. elves, trolls, dwarves, orcs), can be male or female, and their appearance can be adjusted based on pre-set options. A player can have more than one avatar and can play different races or genders, and there are even options in the game for players to change the race and gender of their established avatars. Because of the wide variety of appearances of avatars and the ease with which they are changed for a single player, a certain amount of identity play is licensed in the game world (which will be described further in later sections).

The game itself is a highly immersive open world style game with a high social factor; the game is designed so that players must cooperate with both known and unknown others in order to achieve in-game goals. Players are given quests in the game, some of which can be completed alone but many of which require the player to enlist the help of others. To cooperate, players interact in and around the game using a number of linguistic modes, including but not limited to: in-game text chat rooms, in-game gestures or actions by their avatars, private messages between individual players, and third-party voice chat platforms (e.g. Ventrilo, TeamSpeak). Because of the amount of interaction with others involved in playing the game, relationships often form between players who have never met each other outside of the game context. Players form guilds, or social collectives, which can be organized around a number of factors. Guilds can be made up of friends from outside of the game, employees at the same workplace, ingame acquaintances with similar playing styles and objectives, or complete strangers who band together for a common goal.

The bonds between players, especially those in tight-knit guilds, can be very strong - and this has an effect on deception practices and the detection of deception. To conduct this study, I had to be part of the community to truly understand the ramifications for deception; to this effect, I conducted a long-term study involving high researcher involvement.

\section{The Study}

Data and observations for this work come from a five-year participant-observation ethnography of a community of World of Warcraft players. The ethnography was conducted from June 2007 through August 2012; the objective of the ethnography was to discover how players manage multimodal communication in the game's highly immersive, social environment. To understand how members of this community use language and multimodal communication

\footnotetext{
${ }^{1}$ Source: http://www.mmodata.net
} 
in mundane situations, I had to be part of an active guild and be a competent player in order to be trusted by the other players to have access to all of the language and interactions. To this end, I spent at least ten hours every week as a member of a guild participating in a variety of in-game activities as an active player and sometimes in leadership roles.

Two sources of linguistic data were used for this work in particular: text chat and individual interviews on voice chat. Text chat data were collected using the /chatlog function built into World of Warcraft, which automatically logged all text chat that I was able to view in a text file on my computer. These text chat files contain both mundane interactions in the game world as well as occasional interview-style conversations that I had with players on particular topics of interest. Players in the guild were aware of my research project and data collection and consented to have the logs taken, and identifying information was removed from these logs in post-hoc processing and all avatar names were changed.

Interviews were conducted with fifteen active members of the guild in which my research was situated. The ages of interview participants ranged from 18 to 42 . These interviews were conducted one-on-one over the voice chat program Ventrilo, recorded, and transcribed. In these interviews, players were able to talk to me about their experiences in the game world, and I was able to ask them questions about their gaming history and behaviors. All names and identifying traits have been changed.

Additionally, I kept an ethnographic notebook in which I recorded my observations of major events during the ethnography. These events included occurrences of deception, which I then corroborated with logs from text chat and interview responses from participants involved in these events.

Transcription conventions follow the conventions used in the field of discourse analysis. Lines from text chat are represented in this text using the Courier New typeface and in colors analogous to the way the text originally appeared in the game's interface; for instance, the text of private messages (or 'whispers') from the game appear in a pink font. Interview text appears in indented sections with the name of the speaker offset to the left (either the name of the interviewee or the initials of the interviewer, LBC). When players are being quoted in the text, replaced names and information appear in \{curly brackets\} and any explanatory information added post-hoc for the ease of readers appears in [square brackets].

\section{Deception in World of Warcraft}

Dmitri Williams (2010) urges researchers of virtual worlds to remember the mapping principle, which states that virtual worlds and the "real world" may be contextually similar but differ in important ways that can affect the accuracy of behavioral predictions. Deception in World of Warcraft is one of the cases where the consequences are different due to the parameters of the virtual world. Because of the number of avatar customization options discussed in the introduction to the game above, some identity play and deception is licensed in the community as part of the game experience. MacCallum-Stewart (2008) describes the avatar in World of Warcraft as an object owned by the player rather than an extension of the self - a mindset that players are used to, having had avatar appearance and gender imposed on them in single-player games where there are few or no options for customizing the appearance of the player's avatar. According to MacCallum-Stewart, because the avatar is an owned object, the identity of the avatar is not seen as a reflection of the identity of the player - just as it is reasonable to expect that an elf avatar is not being played by an elf player, it is similarly reasonable to expect that a female avatar in the game might be piloted by a player who is not female. Playing with identity in this way is licensed and expected in the game world, whereas a person in the physical world attempting to portray themselves as a gender they are not may be (for most people) at least unusual, and at worst looked upon with suspicion. However, there are still cases in the 
virtual world of World of Warcraft in which deception has consequences similar to those in offline contexts; I will explore this spectrum of deception in this work.

The cases that I will present in this work involve gender deception - for example, physical males attempting to portray themselves as females in an online context beyond the use of a female avatar. Most of these cases involve men portraying themselves as women, but women do attempt to portray themselves as men. Most of the examples encountered during my ethnography and detailed in this work ended in the deception being detected (whether the deceiver admitted their deception attempt, or whether others detected the attempt as inauthentic), with the exception of one deception attempt revealed to me by a player long after the incident occurred. Because of the numerous cues that deceivers must attend to in their portrayals, they often accidentally reveal clues about their deception (Ekman, 1992), and these clues can take many forms in many different modes. In their discussion of face-to-face interaction, DePaulo and colleagues (2003) suggest that, for example, a deceiver may reveal their deception through visual cues (e.g. fidgeting), verbal cues (hesitations, vocal pitch), and speech errors (slips of the tongue). Once any of these cues are perceived, other interlocutors engage in a questioning process to determine the extent of the deception (Buller \& Burgoon, 1996).

In non-face-to-face mediums, the availability of these cues is reduced and can force heavy reliance on certain modes. Giordano and colleagues (2007) describe the decreased availability of visual cues in telephone conversations, as well as the elimination of verbal and vocal cues in text-based communication like instant messaging. Early studies in deception in computer-mediated communication suggested that the lack of visual and vocal cues lead to significantly lower deception detection accuracy (e.g. Giordano \& George, 2005; Marett \& George 2004). However, later work suggested that since meaningful interactions often take place using multiple modes and mediums, the compilation of cues in many modes may lead to better detection of deception (Giordano \& Tilley, 2006; Giordano et al., 2008; Cooper \& Kahai, 2003). However, an alternate theory suggests that the oversaturation of an interaction many modes can serve to distract interlocutors from a reliable indicator of deception; in the words of George and Marett (2008):

The combination of a less rich environment and more voluminous amount of submitted information (both deceptive and otherwise) can easily drown out a lone deceiver. Unless a group member fortuitously notices a suspicious indicator or discrepancy during the conversation or has a pre-existing suspicion about one of the other members, a deceiver with a convincing story can thrive within a computermediated context. (p.301)

With more experience interacting in a particular medium, users may be better able to detect deception because they have a large body of experience with similar cases and know which warning signs to look for (Carlson \& Zmud, 1999). In summary, while a deceiver may be able to portray an inauthentic identity in a computer-mediated context with more ease because of the different sets of cues available, the experienced members of a community may be able to better detect deception because they have developed strategies for doing so. In World of Warcraft, these strategies involve obtaining information and cues from a number of modes of communication, and the attention given to these cues increases as players form stronger bonds of friendship.

\section{Three Types of Deception in World of Warcraft}

In this work I will deal with three specific themes of identity deception, which all show different manifestations of deception in the community. First, I will discuss a more mundane example, in which players portray an inauthentic identity in text only - sometimes intentionally and sometimes unintentionally - but with no intention of attempting to build a relationship with the other interlocutors based on this deceptive identity. This is the type of deception 
which is more acceptable in the virtual world. Second, I will discuss identity deception in which the speaker employs avoidance tactics to deceive others by avoiding the use of modes which would complicate their deception and add more cues into the multimodal ensemble. Finally, I will discuss two instances where the intentional identity deception extended to modes beyond the game and involved large-scale deception in multiple modes, and which resulted in consequences similar to those of deception in offline contexts. In each case, I will discuss how the deception was detected by members of the community.

It is important to note the relative rarity of these occurrences in a gaming community like World of Warcraft - with the relative anonymity of online communication, it is theoretically possible (and, in fact, common according to work done by researchers such as Turkle (1997)) that any person may be able to take on any persona they wish because of the lack of physical cues. However, aside from a few isolated cases, identity deception is far from the norm in this online community - in fact, the cases depicted in this chapter represent the sum total of deceptions that I observed in my five-year ethnography, and several are from word-of-mouth only. Furthermore, those who do attempt to deceive others are usually quickly discovered through linguistic cues. By analyzing these cases of deception, I do not intend to state that deception happens frequently; on the contrary, I wish to show the few cases of it that I have encountered and the strategies that participants used to see through the deception as an illustration of just how closely participants attend to authenticity in this particular context.

\section{Small-Scale Deception in Text}

In this section, I will describe how players may deceive others (usually unknown players from outside of the community or guild) in text-based modes, whether they do so purposefully or inadvertently. This is the most common type of deception and also the most accepted in the community. The purposeful small-scale deception was reported mostly by female players in this study (myself included), who avoided referring to themselves as female around unknown others, especially when those unknown others were engaging in particularly sexist or masculinist talk. A similar phenomenon has been reported by Nardi (2010) in her discussion of how the language used by men in World of Warcraft can cause women to hide their identities in order to avoid becoming targets of such language. The female players in this study often spoke of having reservations about unknown players finding out they were female and using that as an opportunity to harass them. These players, for example, would not correct others when they used male pronouns, and would purposefully adopt "neutral" language when using text chat in order to avoid scrutiny. Examples of "neutral" language cited by the women interviewed for this study were: lack of emoticons, use of shortened or clipped phrases and words, and not expressing opinions. In all of these cases, players sought to reduce the amount of text they produced in order to limit the amount of opportunities others had to detect their female gender identity.

The use of emoticons, opinionated language, and longer phrases caused Walter, who played a female avatar named Joannis, to report that his writing style often caused unintended confusion regarding his physical gender. Speaking about this phenomenon, Walter described how some other players misread his gender based on "feminine" cues such as hedging, emoticons, acronyms, and politeness:

Walter: $\quad[\ldots]$ my writing style is very feminine in the way it comes off. You know, it's very kind of neutral but it tends feminine. And I've actually heard that from other people on [a forum] and I always thought it was kind of interesting. But I had one person say that you know "if I didn't know better I'd say that you were a woman by the way you write". And [...] when I type and chat and do the same kind of thing it's all the same, I think maybe that I would come off as a woman by the way I type and then I say "oh you know I'm a 
guy" and they're like "Oh! I never saw that coming. I thought you were a woman by the way you type".

LBC: $\quad$ What is it about your typing style that you think or that other people have said that is particularly feminine?

Walter: $\quad$ Um: [...] there's a lot of 'I think', 'I feel', kind of very soft wording in it, you know. Very, 'this is the way I feel', that 'I felt that I've done' - like that kind of writing. [...] I tend to put a lot of LOLs and smiley faces and stuff in my chat so I think maybe that's why it comes across. And I've never been overly crude or your typical male archetypes in chat just because that's just not the way I operate. So I think that's a lot of where that comes from too.

Some players had other motivations for concealing their gender identity. Azhure, a male player who played exclusively female characters, talked about this issue, saying that he wished "people wouldn't make a big deal about gender". I asked him if he would intentionally deceive other players about his gender, and he responded at length:

Azhure: $\quad$ Not necessarily. I just don't think it matters. What do you care if you're trying to kill a guy for a quest, and the person helping you, what does it matter if that person is male or female? [...] And if someone's talking to me, trying to be my friend, just because they think I'm a woman, and then they learn I'm not and stop talking to me, they obviously have other motivations for talking to me besides being friends. I just want to play the game, not have to defend my choices or my identity. It's not like I actively try to pretend I'm a girl or something, I just try not to make it a big priority in my friendships.

When I asked what he would do when someone directly asked about his gender, Azhure responded that he would "avoid the issue", or "turn the question around, like, why does that matter to you?" Azhure, therefore, used the modal affordances of the text chat medium - specifically the lack of cues such as physical appearance or pitch of voice - to deprioritize gender in budding friendships. This was not through malicious intent, but rather a desire to avoid the problematization of avatar gender and identity. In the guild and with his close friends, Azhure never attempted to portray himself as a woman, always being straightforward about his male gender and correcting others' incorrect use of pronouns when they happened.

Either intentionally or unintentionally, there are a number of stylistic resources available in text chat that can lead to a person's gender being interpreted one way or another based on the reader's preconceived notions of that type of style. Since a textual style including more hedges and emoticons is often associated with females, those men who incorporate these styles into their discourse will often be read as women (corroborated by the work o Thomson and Murachver, 2001). When there is some question about the gender of the player or the gender is unclear, players will either ask directly whether the player is male or female, or seek other cues about gender in other modes of communication. Typically, the mode that players will use for detection of deception in writing style is voice chat. Textual style may be easier to manipulate based on the ability to compose and edit a textual utterance before sending the message, but verbal style - including the pitch of one's voice - is a clear indicator of gender. Often voice chat is the next step for verification of authenticity of gender.

\section{Deception by Avoidance}

When seeking to confirm the authenticity of an interlocutor's gender performance, players will often turn to voice chat. Azhure, who above admitted to intentionally masking his gender in text, never shied away from revealing his 
male gender identity and male-sounding voice on voice chat. Walter, too, experienced some other players being shocked at the mismatch between his male voice and his written style that indexed female identity in discourse.

These examples show how authenticity can be verified using voice chat, and since voice chat is a primary means of communication in more advanced gaming communities, those players who wish to continue to engage in deception will have to consider their use of voice chat some point. There are two strategies for continuing deception: some players attempt to disguise voice-based cues in voice chat, which I will discuss in the next section of this article, but the most common strategy for continued deception is to avoid the use of voice chat altogether (often in conspicuous ways). This reflects other research in computer-mediated contexts that suggest that interlocutors are more likely to adopt avoidance strategies in computer-mediated negotiations than they would in face-to-face interactions (e.g. Dorado et al., 2002). In my ethnographic experience, which was corroborated in the interviews, the particular avoidance strategy that many players used was to say that they did not have a working microphone to use voice chat. By blaming the technology, these players sought to deflect scrutiny of their gender performance and avoid the use of the voice because the voice carries cues to gender (most notably, pitch). These players would freely continue to talk in the text chat mode, but avoid voice chat altogether.

Avoidance of voice chat is often done with unknown players from outside of the core community or guild until a stronger bond was made between these players. Female players such as Haley would often avoid talking on voice chat with unknown others because of the fear of harassment for their gender.

Haley: I probably wouldn't willingly talk on voice with people outside of our guild, either, unless, say, I knew them and they were friends or something like that.

LBC: $\quad[\ldots]$ Why wouldn't you be willing to talk to strangers?

Haley: I think it depends on the situation, I think for me as a girl there's definitely the factor of there's some creepy guys out there. [laugh] And I don't wanna deal with that at all, or even be put in that situation, and so it'd be easier just to chat first in text with them for a while.

Most players agreed with Haley in their interviews - after talking with someone for a while and establishing a friendship, it is expected that they will interact using voice chat. Therefore, as bonds between players strengthened, players generally found an interlocutor's continued avoidance of voice chat to be suspicious. Two players in the study, Laira and Rufus, both cited the case of a player named Esmerelle who claimed to be a female but never spoke on voice chat (although she was often present in the voice chat channels listening to others). Esmerelle engaged in flirtatious conversations with male players often but entirely avoided moving to interactions in non-text modes by claiming a lack of a working microphone. This behavior cast doubt on Esmerelle's gender, and both Laira and Rufus reported that they would scrutinize Esmerelle's performances in other modes of communication such as trying to 'trap' her with lack of knowledge about female-related topics. They were amused by Esmerelle's continued flirting and were convinced that Esmerelle was really a "he" and referred to her that way.

The one case of identity deception that went undetected was the experience of Rawka, who used avoidance strategies and deflection to successfully conceal his male gender identity. During his interview, Rawka described his history as a "mailbox dancer", or a person who engaged in in-game sex chat in return for virtual gold. Rawka pretended to be a female and chatted with strangers under this guise in order to raise the funds to pay for expensive in-game items. I asked him about his strategies for trying to deceive others and he referred to using avatar gestures:

LBC: $\quad$ Did you have any language strategies, or different ways that you would talk or type while doing this, to try to be ambiguous or-? 
Rawka: I tried to use the emote system [having characters perform actions] as much as possible, it was in-game so if you use /kiss or /lick people really liked that one. I would try to use the emote system as much as possible, so it was more of like a role-playing scenario where it was actually happening.

By using the visual mode and the gestures of his female avatar, Rawka focused the interaction on the virtual female body rather than the text or language that he was producing. By using this method, he deflected attention from his language performance. Rawka also described what happened when his customers brought up the idea of talking in voice chat.

LBC: $\quad$ Did any of those people ever ask you to like, get on Vent [a voice chat platform] and talk to them?

Rawka: All the time.

LBC: $\quad$ And what would you tell them?

Rawka: I told them that that would be a higher charge. [laughing]

LBC: [laugh] And what did they say?

Rawka: I would never get into Vent with them though, I mean it would never go that far. I would always make it like, something obscene that they didn't wanna pay. I would never get into Vent with anybody. Just 'cause that to me is kind of, not that what I was doing was okay, don't get me wrong [...] but I just, I probably would not do it, because it could potentially be dangerous.

Rawka said that when things got to the point that his interlocutors demanded voice chat with him (or more threatening information like address and phone number), he would use in-game tools to block the other person from communicating with him and cut off all future contact. That was getting too close, he said, and he did not feel comfortable continuing the interaction because he did not want to be discovered to be committing deception.

Rawka's mailbox dancing days happened years before the interview, when the World of Warcraft community was smaller. At the time of his interview, the mailbox dancing phenomenon had mostly disappeared for two reasons: first, it is much easier to obtain in-game currency in the current versions of the game, and second, most players believe that the person behind the sexy talking mailbox dancing female avatar is actually a male. When asking others about the phenomenon, they cited the commonly accepted practice of playing an avatar of a different gender than the player. Laira, a veteran World of Warcraft player, said that "people figure it out pretty quickly when it's common to hear these very male voices attached to dainty female characters".

Even though the notion of deceptive identity play is widespread in the community, some players still attempt to engage in identity deception on a broader scale. To do so, they must orchestrate their performance in all of the available modes of communication, both in-game and out of the game. During the time of my ethnography, two cases of large-scale deception occurred in which players attempted to engage in identity deception in game-external modes.

\section{Large-Scale Deception in Game-external Modes}

Larger-scale deception requires innovative multimodal communication and thus allows for more opportunities for the deceiver to falter in their inauthentic portrayal. In this section, I will describe the two cases of this phenomenon that I encountered during my ethnography. 
The modal avoidance strategy described in the previous section is well-known by the players of the game, and any person who reported themselves to be a female but who refused to talk on voice chat had their gender identity put into immediate question. Although this avoidance alone could not be solid proof of deception, it was often the first sign and caused players to look for other cues. One instance of this during my ethnography involved a player named Filly. Filly joined the guild in early 2009, reported herself as female in the introductory post on the guild forums, and referred to herself as female. She became close to Natholis, a well-connected and popular player in the group, and the two engaged in what seemed to be the beginnings of romance. They shared photographs of each other, connected on Facebook, and communicated by SMS, or text messaging, However, several of Natholis's friends were concerned - Filly never used voice chat and claimed that she did not own a microphone, consistently using this excuse even with Natholis. This was not sufficient proof for Natholis to believe that Filly was deceiving him, however, and several of Natholis's friends engaged in an attempt to trap Filly in her deception. Jahaerys, a close friend of Natholis and fellow guild member, described the incident:

Jahaerys: $\quad$ There was one instance of it, um, about a year into me playing after I joined [the guild], I believe. \{Natholis\} had become friends with a girl, um, that the rest of us assumed was a guy, and he didn't believe us, but then when we called that person out, they sort of disappeared from the game.

LBC: $\quad$ I remember-

Jahaerys: $\quad$ Like, she sent him pictures and stuff, and I was like, "Uh, that looks more like a model than an actual person?"

LBC: $\quad$ Uh- do you remember the pictures? I think I remember them.

Jahaerys: $\quad[\ldots]$ The picture kinda looked like a setup for like, the photo frame, when you buy one and it's already got somebody in it.

LBC: $\quad$ What else about that person do you remember, kind of tipped you off that this might not have been a female?

Jahaerys: $\quad$ Just how she always had an excuse for like, "Oh, hey, wanna get on [voice chat]?" or something, and she'd be like, "Oh, I don't have a microphone." And we're like, "You could just download it, and don't have to say anything." And she would never, it always seemed suspicious. There was always an excuse for why she couldn't do something.

LBC: $\quad$ Wasn't $\{$ Natholis $\}$ texting her too, for a while?

Jahaerys: $\quad$ Yeah, and then like one day, she stopped responding.

LBC: $\quad$ Did he ever try to call her, or did anybody ever try to call her, do you know?

Jahaerys: I don't know. I think he gave the player \{Paetrik\} her phone number, but I don't think anyone ever called her, at least not that I know of.

Jahaerys cites a number of resources that he used to try to convince Natholis of Filly's deception, including the avoidance of voice chat, suspiciously perfect pictures posted in the guild's forums, and a telephone call to her cell phone number. Paetrik, who was given Filly's phone number, told me about this telephone call in a private message in text chat.

Paetrik whispers: so I called $\{$ Filly\}

You whisper: Oh? What happened? 


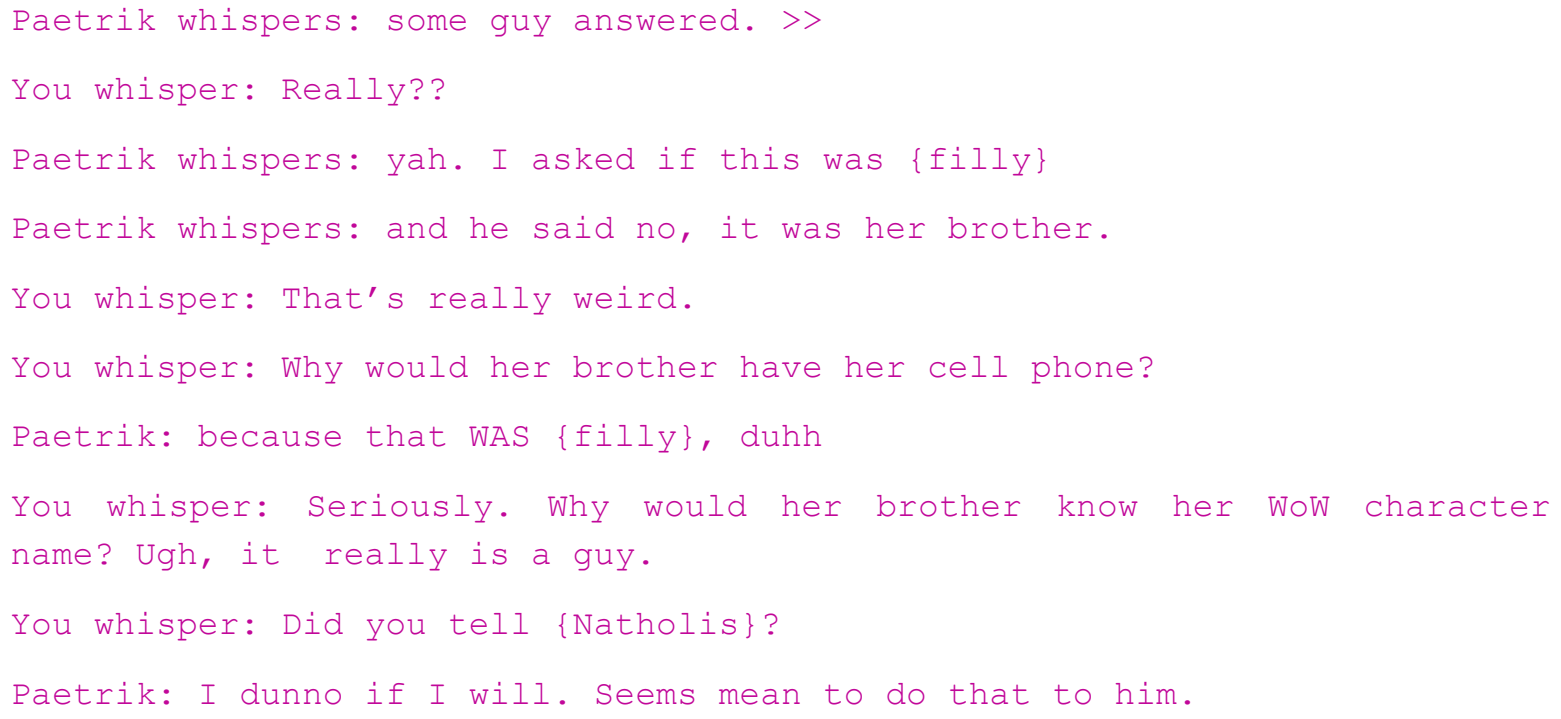

The suspicious cues about Filly were drawn from a variety of multimodal resources: her lack of participation in the voice chat mode, her photographs posted on the guild's forums that seemed too perfect (whether too much like a model, or too perfect), the sudden lack of response to text messages, and the male voice that answered a telephone call.

As part of her ruse, Filly avoided voice chat and chatted only by text-based modes including SMS messaging; however, there are ways to still use voice modes like voice chat deceptively. One of these is by using a program called a "voice changer" that can modify the voice of the speaker. One popular voice chat platform, Ventrilo, has a voice changing setting built in; some players cited friends and guildmates who would use the voice changer for humor, to play with the options available and to experiment with voice quality. In these cases, the voice changing option was not used to deceive because all listeners knew the identity of the speaker. Paetrik, who was involved in the investigation of Filly, had an experience that revealed a more insidious use of a voice changer - an individual used a voice changer from the beginning of the interaction, therefore never allowing others to hear the 'real' voice of the player. After we encountered this deceptive player in World of Warcraft, Paetrik told me the story of how he had been deceived.

Sharky and Paetrik met in Final Fantasy XI, another MMORPG that was quite popular before World of Warcraft was released, and they also played World of Warcraft together. Sharky claimed to be female, and talked to Paetrik often in the game; soon, Paetrik began to experience romantic feelings for Sharky. Paetrik asked to talk to Sharky on voice chat and she logged in and talked to Paetrik with a distinctly female voice. Paetrik did note that her voice sounded a little robotic, and Sharky's excuse was that she was using a low-quality microphone that was built into her webcam. Relieved to have Sharky's gender confirmed, Paetrik felt comfortable expressing his romantic interest which Sharky returned. Several months into their relationship, Paetrik's friend and fellow guildmate Mork was talking to Sharky on voice chat when something happened. Mork told me later about it:

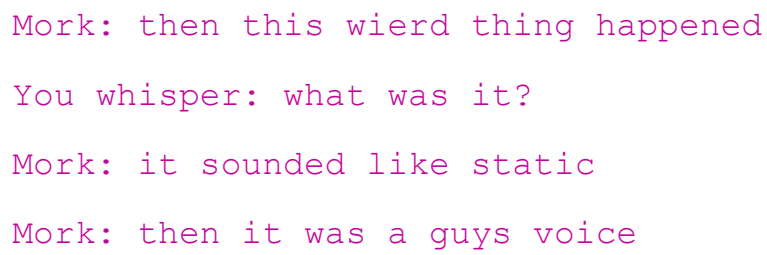

In Mork's estimation, Sharky had been using a voice changer and it had experienced some sort of error and stopped working in the middle of his sentence, revealing Sharky's actual voice which was definitely male-sounding. Mork 
told Paetrik what had happened and Paetrik confronted Sharky about it. Sharky admitted to using a voice-changer and deceiving Paetrik.

Paetrik said that Sharky tried to contact him several times after this revelation in an attempt to continue the friendship (including once during my ethnography), which made Paetrik uncomfortable. Paetrik had no interest in remaining in contact with Sharky after this incident. Each time he talked about Sharky, Paetrik expressed disgust and disdain at this behavior, and mostly embarrassment that he could be fooled so badly. Paetrik had, in fact, been victimized by Sharky's deception. Sharky's deception provides an interesting comparison with Rawka's - Paetrik and Sharky had a meaningful relationship and talked to each other using a mode which is supposed to reveal the identity of the player. Furthermore, Paetrik had trusted Sharky enough to admit interest in a romantic relationship, and that trust had been violated by the revelation that Sharky's identity had been inauthentic. Rawka's contacts were short-lived and never went to a meaningful level because he cut off contact before that could happen. This comparison shows the role of relationships in deception - most players agree that deception is licensed in short-term interactions when no friendship is being developed. Women who hide their gender to protect themselves from harassment by unknown others are accepted because they are not developing meaningful relationships with these people; this viewpoint can extend to the case of Rawka, who was not attempting to develop a relationship but rather sell a service. It is when connections are formed and friendships are made that deception becomes problematic, both for the deceiver and the deceived, and when the consequences more closely mirror those that happen in offline contexts.

While stories of deception like the ones discussed above seem to get a lot of air time both in the media and in the community, they are the exception rather than the rule. As Baym (2011) has asserted, people in online communities tend to look for meaningful connections and authenticity rather than a chance to experiment with identity. The rarity of large-scale deception cases in my five-year ethnography provides evidence that these incidents are not as prolific as they may seem. However, members of the community are still wary of this type of deception happening to them (perhaps because of how much they are talked about), and this can cause consequences for interactions on a broad scale.

\section{Unintended Consequences}

In some cases, the wariness about deception, particularly about gender, can have unintended consequences. In general, these consequences include an increased pressure on women to 'prove' their identity, in ways that could sometimes be uncomfortable. In guilds like the one I studied, where women were active members, there were restrictions in place to prevent women from being uncomfortable about revealing their gender. However, not all guilds had these rules in place, and not all players followed the rules. One case that illustrates this phenomenon is the story of a player named Roxanne.

Roxanne was a member of another guild that focused on the game activity of roleplaying, or the act of interacting inside the game environment as the avatar with an in-game backstory; specifically, this guild catered to players who enjoyed erotic roleplaying (as a hobby, not for in-game currency like Rawka and other mailbox dancers). Roxanne sometimes joined our guild for events because she was friends with Laira, a popular guild member. As reported by Laira and several other players in my study, there was a considerable controversy surrounding Roxanne because of uncertainty about her gender. Rumor had it that Roxanne was a male player masquerading as a female - the evidence Laira reported for this was similar to that used to implicate Filly as a male player, such as "She refuses to talk on voice chat" and "The pictures she posted online are too sexy to be real". However, because of players like Rawka who would pose as women to solicit in-game currency, there existed a commonly-held belief that only men engaged in this type of erotic roleplay and they usually did so while posing as women. Roxanne talked to me in- 
game once about how she felt that some of the players in my guild were harassing her by questioning her gender and her erotic roleplaying.

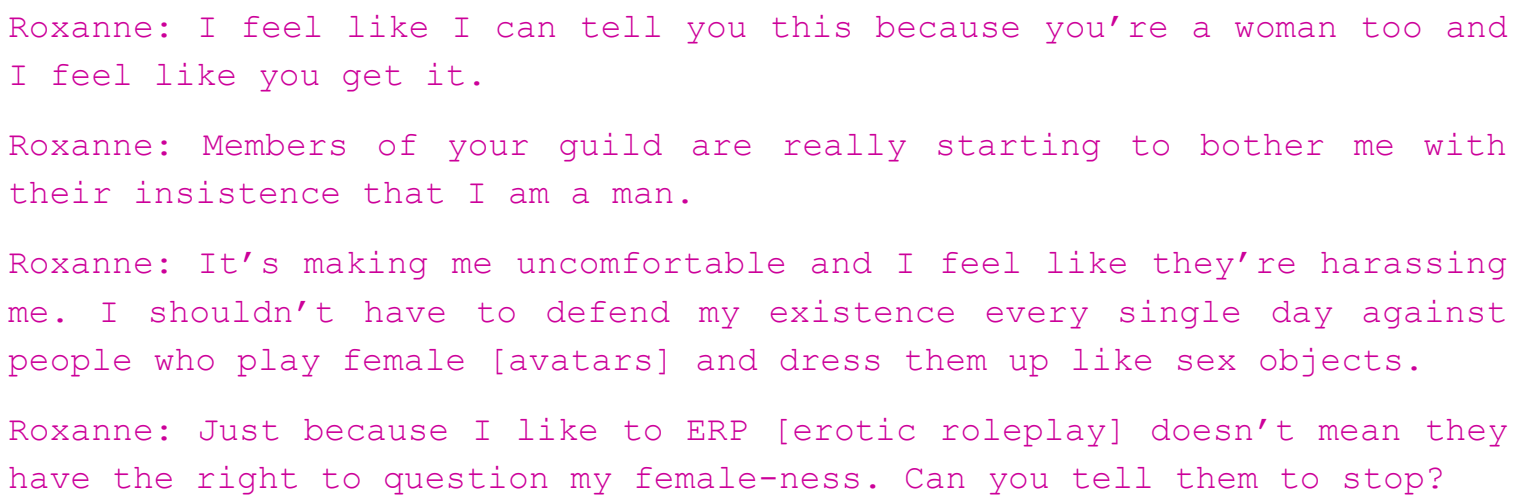

Roxanne's concern went beyond the annoyance of being questioned about her gender - she was dismayed that she had to defend her identity against people who regularly appropriated her identity for pleasure. Much later, during a chat conversation in 2012, Laira described the proof she had obtained of Roxanne's gender identity:

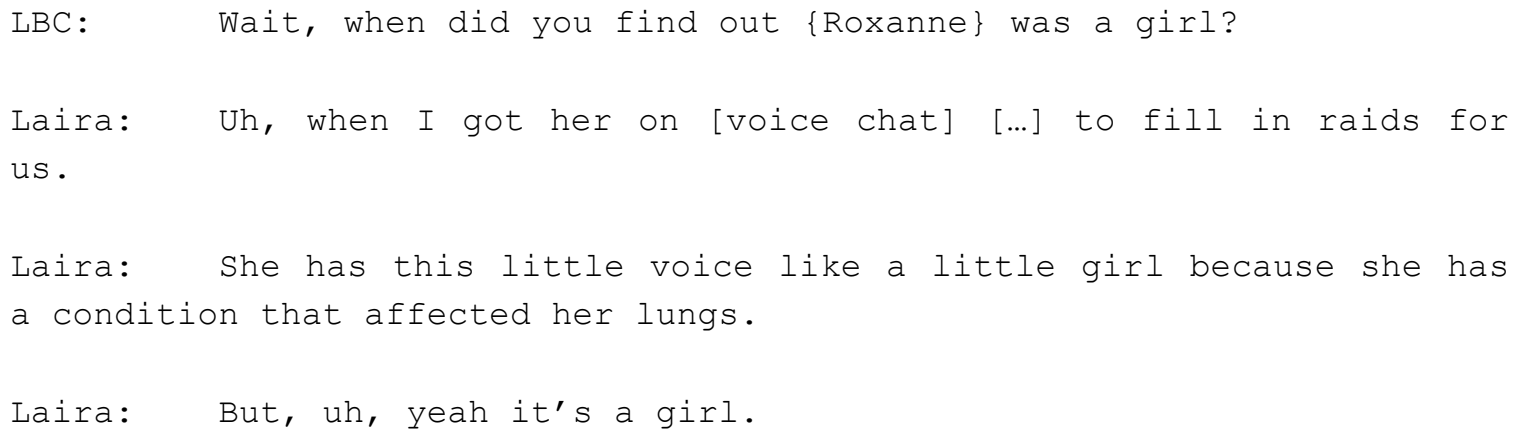

For Laira, hearing Roxanne's voice on Ventrilo confirmed Roxanne's gender identity. Roxanne chose not to speak on Ventrilo because of her vocal condition; however, since a common tactic for those intending to deceive others about their identity is to avoid use of Ventrilo, Roxanne's avoidance of Ventrilo caused her gender identity to be questioned. When players matched Roxanne's reluctance to use voice chat in combination of her outward expressions of eroticism by participating in erotic roleplay, the conclusion that she was being deceptive about her gender seemed apparent to most players. Even Laira, who heard Roxanne's voice and deduced that she was female, continued to refer to Roxanne as "it" rather than using feminine pronouns; in fact, Laira referred to Roxanne the same way she referred to Esmerelle, for whom she never obtained solid proof of gender identity. Roxanne was reduced to "it" for sending cues about identity deception, even though she had been confirmed to be a female.

The case of Roxanne reveals the contradictions in multimodal communication and deception that women in World of Warcraft environment must navigate - women are always subjected to questioning about their gender identity, yet revealing oneself as a woman may open a player up to unwanted contact and harassment based on her gender (Nardi, 2010). If a woman talks on Ventrilo and receives unwanted advances from male players, she may be reluctant to talk on Ventrilo again (as Haley said regarding "creepy guys" above). To prove her gender identity in cases where she wishes to do so, a woman may post a photograph of herself as proof of her identity; however a woman posting a photograph deemed "sexy" may bring her gender identity into question, as Filly's photographs did. Women are subjected to unwanted romantic and erotic advances by men, but when a woman engages in erotic or sexual behavior, she is often either assumed to not be women or shamed for her erotic interests. 
This problematic situation for women in World of Warcraft complicates the picture of deception detection, because in order to have a pleasant playing experience some women must avoid revealing their gender. This is not unique to women; similar situations happen for people of Chinese ethnicity (see, e.g. Nakamura (2009) and Steinkuehler (2006) on the racialization of labor and the association between Chinese players and illicit behaviors) and for those who identify as non-heterosexual (see, e.g. Collister, 2013, p. 176-177). This would provide a difficult situation to overcome for any party attempting to apply a unilateral detection algorithm, as it would incorrectly identify as deceivers these people for whom these strategies are a safety net.

\section{Summary}

In this section, I described three types of deceptive behaviors in World of Warcraft and the multimodal strategies that players used to discern deception in their community. Table 1 summarizes these findings.

\begin{tabular}{|l|l|}
\hline Deceptive Behavior & Strategies for Detection \\
\hline $\begin{array}{l}\text { Small-scale Text-based Deception - avoiding pronouns } \\
\text { in self-reference, using gender-stereotypical features } \\
\text { (e.g. emoticons). }\end{array}$ & Ask directly about gender or move to voice chat \\
\hline $\begin{array}{l}\text { Avoidance-based Deception - avoiding modes in which } \\
\text { deception is easier to detect }\end{array}$ & $\begin{array}{l}\text { Continue to develop relationship, scrutinize other modes } \\
\text { for cues (e.g. photos, text chat) }\end{array}$ \\
\hline $\begin{array}{l}\text { Large-scale External Mode Deception - using } \\
\text { technological affordances to enable deception in } \\
\text { multiple modes over a long period of time }\end{array}$ & $\begin{array}{l}\text { Move away from deception-enabling technology (e.g. } \\
\text { microphones), interact with person outside of game, use } \\
\text { third parties }\end{array}$ \\
\hline
\end{tabular}

Table 1: Summary of deceptive behaviors and detection strategies.

Some deception in World of Warcraft was in self-defense, as was the case for women who hid their gender identities to avoid harassment. Some was unintentional, such as Walter, whose writing style had many features that were seen as feminine. Other cases of deception were for a purpose, whether it was Azhure trying to fight gender norms in friendship formation or Rawka pretending to be female to make money. Yet others were malicious in their deception, going to great lengths to fool other players and make them uncomfortable.

Some deception was licensed in the community based on the affordances of the virtual world. A mismatch between gender identity of a player and their avatar was acceptable, as was avoiding gender assignment in interactions with unknown others. Once interactions become more than fleeting and one begins to make friends in the game, then the deception is not licensed any longer; one must 'come clean' with others or risk alienation and suspicion. Those players who avoid certain modes, particularly those with a connection to one's physical self and identity, are seen as suspicious and possibly not worthy of a closer relationship. Rawka blocked future interactions in order to avoid having to admit his deception; yet, relationships were destroyed by players not admitting their deceptive behaviors, as in the cases of Filly and Sharky. These players who engage in deception by avoidance are tolerated but 
investigated and possibly mocked; those who go beyond avoidance and actively work to deceive in all linguistic modes are shunned. These outcomes are more similar to consequences of deception in offline contexts.

The observable pattern for members of the community is in their attempts to detect deception. All of the participants interviewed used the multimodal tools available to them, gradually moving to modes that are closer to face-to-face interaction to observe more clues about the identity of their interlocutors. Voice chat reveals the speaking patterns and verbal characteristics of interlocutors, and cues such as pitch may reveal the identity of the speaker. Furthermore, voice chat is a more synchronous medium than text chat, allowing less time for speakers to compose their utterances and edit them. These aspects of the voice chat mode make it more likely for identity deception to be detected, and explain the desire for deceivers to avoid this mode altogether if possible. When more proof is needed to detect deception, players look to more offline modes such as phone calls to gather additional cues. This particular mode backfired for Sharky, who was able to continue his deception in the text-based SMS mode but revealed his male voice when answering a phone call to the same telephone number. The final step is ostensibly that a player might attempt to meet face-to-face with another player in order to detect their deception; however, no examples of any deception attempt going this far occurred during my ethnography.

I have also shown how the concern about deception in the community has led to negative consequences for some of its members, particularly those who are in the minority identity groups. Women, a minority in the game's population, often have their gender identity appropriated by those wishing to deceive others; this can lead to an increased pressure on women to 'prove' themselves in increasingly uncomfortable ways. Any individual attempting to detect deception in a community like this must be wary of these kinds of outcomes; the strategies used to detect deception may cause discomfort for some players when applied too liberally.

\section{Conclusion}

In this work, I have shown how deception is viewed in the virtual world of the online game World of Warcraft. I detailed a few cases of identity deception, from the mundane (mistaking someone's gender based on cues in their typed text) to the exceptional (using a voice changer over a period of months to actively deceive another into a relationship). In each of these cases, I have shown how mode of communication is a vital component in either perpetuating or untangling the deception. The different modes of communication that these players use are not just tools to make interactions easier in fast-paced gaming contexts, but rather a resource used in the creation and maintenance of relationships and identities. In minor cases of unintentional textual deception, an interaction with the other using voice chat can easily reveal the speaker's identity. For most players, this is the norm of interaction and identity - recalling Williams's (2010) mapping principle, some deception is licensed within the bounds of the game world because of the different rules that apply within the medium. It is acceptable for a player to withhold their gender identity from unknown others because they may never interact with these other players again. Identity play can even be thought of as part of the fun of interacting with so many random strangers in this large community. However, once those interactions extend beyond the medium and become important outside of the game, then deception is not licensed.

This work also gives insight into how to assess an individual's behavior in a virtual world context. Investigating virtual world interactions can give important clues about an individual engaging in deception; however, this relies on the investigator having an understanding of the norms of interaction in the community they are studying as well as competency in the modes of communication. If an investigator is looking to an individual's presence in a virtual world to gather intelligence about that person, it is important to understand the norms of behavior in these virtual worlds before attempting to derive information about the individual. Some level of deception may be licensed (and 
even embraced) by communities in virtual worlds and, therefore, deception (especially on small scales) may not mean the same thing in the virtual worlds as it does in offline contexts.

Beyond virtual worlds, investigation of multimodal communication in general can be beneficial to those engaging in deception detection. Cues to deception are highly context-dependent and require the investigator to have knowledge of the nuances of communication. When interacting in a multimodal environment, a deceiver must address all of the modes of communication or risk looking suspicious; investigating a suspected deceiver in many modes of communication will yield proof of deception to build the case. Particular scrutiny may be given to those who conspicuously avoid modes of communication which are vital to interactional norms in the community. However, in gathering intelligence about individuals, it is important to take identity into account when looking at interaction patterns; individuals may be engaging in some form of deception to protect themselves from other members of the community. This is particularly pertinent in online contexts where security of one's private information may not always be guaranteed. Therefore, I urge researchers and investigators who are interested in obtaining intelligence in virtual worlds to take into account the parameters of the virtual world they are studying and to recall that different rules exist which result in different behaviors and outcomes. Important information can be gleaned from virtual worlds, but only if that information is appropriately situated in context.

\section{References}

Baym, N. (2010). Personal connections in the digital age. Malden, MA: Polity.

Blizzard Entertainment. (2004). World of Warcraft.

Buller, D. B., \& Burgoon, J. K. (1996). Interpersonal deception theory. Communication Theory, 6(3), 203-242.

http://dx.doi.org/10.1111/j.1468-2885.1996.tb00127.x

Carlson, J., \& Zmud, R. (1999). Channel expansion theory and the experimental nature of media richness perceptions. Academy of Management Journal, 42, 153-170. http://dx.doi.org/10.2307/257090

Collister, L. B. (2013). Multimodality as a sociolinguistic resource: A case study of World of Warcraft. Dissertation, University of Pittsburgh. http://d-scholarship.pitt.edu/18514/

Cooper, R. \& Kahai, S. (2003). Exploring the core concepts of media richness theory: The impact of cue multiplicity and feedback immediacy on decision quality. Journal of Management Information Systems, 20, 263-29

DePaulo, B. M., Lindsay, J. J., Malone, B. E., Muhlenbruck, L., Charlton, K., \& Cooper, H. (2003). Cues to deception. Psychological Bulletin, 129(1), 74-112.

Ekman, P. (1992). Telling lies: Clues to deceit in the marketplace, politics, and marriage. New York: W.W. Norton. George, J. \& Marett, K. (2008). Deceptive communication in e-collaboration. In S. Kelsey \& K. St. Amant (Eds.), Handbook of Research on Computer Mediated Communication. Hershey, PA: Information Science Reference. http://dx.doi.org/10.4018/978-1-59904-863-5.ch023

Giordano, G. A. (2007). The effects of group member experience and task complexity on computer-mediated groups 
facing deception. Dissertation, Florida State University.

Giordano, G. A., Stoner, J. S., Brouer, R. L., \& George, J. F. (2007). The influences of deception and computermediation on dyadic negotiations. Journal of Computer-Mediated Communication, 12(2), 362-383. http://dx.doi.org/10.1111/j.1083-6101.2007.00329.x

Giordano, G. A., Stoner, J. S., Brouer, R. L., \& George, J. F. (2008). Computer mediated negotiations and deception. In S. Kelsey \& K. St. Amant (Eds.), Handbook of Research on Computer Mediated Communication (220-229). Hershey, PA: Information Science Reference. http://dx.doi.org/10.4018/978-1-59904-863-5.ch017

Giordano, G. A. \& Tilley, P. (2006). The effects of computer-mediation, training, and warning on false alarms in an interview setting. Communications of the AIS, 18(6).

Jordan, T. (1999). Cyberpower: The Culture and Politics of Cyberspace and the Internet. New York: Routledge.

MacCallum-Stewart, E. (2008). Real boys carry girly epics: Normalising gender bending in online games.

Eludamos, 2(1).

Marett, K., \& George, J. F. (2004). Deception in the case of one sender and multiple receivers. Group Decision and Negotiation, 13(1), 29-44.

Nakamura, L. (2009). Don't hate the player, hate the game: The racialization of labor in World of Warcraft. Critical Studies in Media Communication, 26(2), 128-144. http://dx.doi.org/10.1080/15295030902860252

Nardi, B. (2010). My life as a night elf priest: An anthropological account of World of Warcraft. Ann Arbor: University of Michigan Press.

Steinkuehler, C. (2006). The mangle of play. Games and Culture, 1(3), 199-213.

http://dx.doi.org/10.1177/1555412006290440

Thomson, R., \& Murachver, T. (2001). Predicting gender from electronic discourse. British Journal of Social Psychology, 40, 193-208. http://dx.doi.org/10.1348/014466601164812

Trend, D. (2001). Reading Digital Culture. Malden, MA: Blackwell Publishing.

Turkle, S. (1997). Tinysex and gender trouble. In Kemp, S. \& Squires, J. (Eds.), Feminisms (516-520). New York: Oxford University Press.

Williams, D. (2010). The mapping principle, and a research framework for virtual worlds. Communication Theory, 20(4), 451-470. http://dx.doi.org/10.1111/j.1468-2885.2010.01371.x 